\title{
Uso del role playing en la enseñanza de mercados eléctricos: ejercitar y comprender el poder de mercado en los mismos.
}

\author{
David Ribó-Pérez ${ }^{a}$, Manuel Alcázar-Ortega ${ }^{\text {a }}$ y Carlos Álvarez-Bel ${ }^{a}$ \\ ${ }^{a}$ Departamento de Ingeniería Eléctrica, Universitat Politècnica de València, Camino de Vera, s/n, \\ edificio 5E, $2^{\mathrm{a}}$ planta. 46022 Valencia (España)
}

\begin{abstract}
Undestanding the functioning of electricity markets in lectures requires the use of software simulators. Due to the impossibility to participate in real markets, it becomes essential to adopt virtual environments to simulate what happens in actual markets. Nevertheless, once designing these simulators, it is common to eliminate scenarios that do not fulfill the perfect economic theory. Thus, these simulators tend to eliminate the cases where market power occurs. In particular, this article describes and anylises a role playing methodology with electricity market simulators in environments characterized by market power. The results and opinions of students are presented to validate the utility and applicability of these type of methodologies.
\end{abstract}

Keywords: market power, electricity markets, role playing

\begin{abstract}
Resumen
La comprensión del funcionamiento de los mercados eléctricos en las aulas requiere el uso de simuladores informáticos dada la imposibilidad de participar en mercados reales, por lo que resulta necesaria la incorporación de entornos virtuales que simulen aquello que ocurre en los mercados reales. Sin embargo, a la hora de diseñar estos simuladores, suelen obviarse aquellos escenarios que no cumplen la teoría económica, lo que elimina una gran mayor parte de los casos donde los fallos de mercado no se tienen en cuenta. En particular, en este artículo se describe y analiza la metodología de role playing en simuladores del mercado eléctrico con condiciones de poder de mercado. Los resultados y opiniones de los estudiantes son presentados para validar la idoneidad y la utilidad de este tipo de metodologías.
\end{abstract}

Palabras clave: poder de mercado, mercados eléctricos, role playing

\section{Introducción}

Las prácticas en el laboratorio resultan una herramienta esencial a la hora de adquirir competencias en el aprendizaje en los campos ingenieriles (Montes Granada, 2004). Estas son utilizadas para afianzar conceptos teóricos presentados en clase de una manera dinámica y participativa. Sin embargo, esto puede resultar complejo. En el caso de las asignaturas asociadas a los sistemas eléctricos de potencia esto ocurre debido a la imposibilidad de 
Uso del role playing en la enseñanza de mercados eléctricos: ejercitar y comprender el poder de mercado en los mismos.

realizar prácticas en entornos reales ya que estos presentan elevados grados de complejidad y criticidad (Alcázar Ortega \& Álvarez Bel, 2018). De igual forma ocurre con las transacciones económicos asociadas a estos. La competencia económica en el mercado de la electricidad tras la liberalización del sector en Europa en la última década del siglo pasado ha sido plasmada en la implementación de complejos mercados y mecanismos de compraventa de la energía. Debido a la imposibilidad de practicar en entornos profesionales, en este campo han de realizarse simulaciones informáticas para ejemplificar la práctica en clase. Por ello, diferentes universidades han planteado herramientas informáticas para simular la participación en mercados eléctricos (Carpio et al., 2007; Contreras, Conejo, de la Torre, \& Munoz, 2002; Guevara-Cedeno, Palma-Behnke, \& Uribe, 2012; Hua, Ngan, \& Fushuan Wen, 2007; Naukkarinen et al., 2017).

Este tipo de aplicaciones ha sido presentado como una herramienta útil para la adquisición de competencias por parte del alumnado. En particular, se desarrollan las siguientes competencias específicas (Guevara-Cedeno et al., 2012):

- Comprensión del diseño del mercado eléctrico y el modo de actuación de cada uno de los agentes

- Implementación de estrategias de operación para participar en mercados eléctricos

- Resolución de problemas de equilibrio de mercado

Sin embargo, estos simuladores suelen utilizarse para modelar mercados que se comportan de forma competitiva, donde los participantes no ejercen poder de mercado. Dicho ésto, el mercado eléctrico ha presentado históricamente elevados grados de concentración y problemas de colusión (Borenstein, Bushneil, \& Knittel, 1999; Borenstein, Bushnell, Kahn, \& Stoft, 1995; Fabra \& Toro, 2005). O recientemente, multas a compañías en el mercado español por reducir estratégicamente la producción para aumentar los precios (CNMC, 2015). Por ello, resulta necesario no utilizar únicamente los simuladores como mercados perfectos, si no también fomentar comportamientos colusivos en ellos, entendiendo como acuerdos colusivos la coordinación del comportamiento empresarial con el fin de subir precios o reducir producción con el fin de aumentar los beneficios. Con el objetivo de mejorar así la comprensión del fallo de mercado asociado al poder de mercado por parte de la oferta o de la demanda. Por ello, se plantea la realización de un ejercicio de role playing en simuladores de mercados eléctricos para experimentar la utilización de poder de mercado.

La comunicación de congreso se estructura de la siguiente forma: la sección 2 muestra los objetivos de la metodología docente descrita en este documento. La sección 3 explica el fundamento teórico de la actividad docente, el simulador utilizado para la dinámica y la dinámica particular de participación en mercados eléctricos. La sección 4 muestra los resultados obtenidos en un caso práctico realizado en un aula y el feedback realizado por el alumnado. Finalmente, las conclusiones del uso de esta metodología docente en el caso de estudio se exponen en la sección 5. 


\section{Objetivos}

Los objetivos del trabajo que se presenta son los siguientes:

- Presentar los resultados de aprendizaje entre el alumnado del uso de técnicas de role playing para exponer su potencial.

- Describir la metodología y simulador utilizados para la realización de prácticas del mercado eléctrico con poder de mercado en entornos docentes.

- Mostrar a los docentes el potencial de la aplicación de metodologías de role playing en el campo de la docencia universitaria.

- Presentar la opinión del alumnado a cerca de esta actividad.

\section{Desarrollo de la innovación}

La innovación se enmarca en la participación de agentes competitivos en los mercados eléctricos. Tras la liberalización de estos desde los años 80 , se han desarrollado diversos tipos de mercado y se ha ido refinando la legislación. Sin embargo, en este tipo de mercados se sigue poniendo énfasis en la necesidad de seguir hacerlos más competitivos. En el presente documento se tratará la participación en mercados mayoristas con un método de fijación de precios marginalista, tal y como se encuentra implementado en la península Ibérica (OMIE, 2019).

\subsection{Fundamento teórico}

\section{Mercado marginalista de electricidad}

El mercado de electricidad funciona como una subasta por ambos lados. Para cada periodo de tiempo (1 hora en el mercado Ibérico) los productores presentan ofertas para vender una cantidad de electricidad a un precio, por su parte, los agentes compradores presentan ofertas para comprar ciertas cantidades de electricidad a un precio determinado. Es entonces, cuando el Operador del Mercado Ibérico de Electricidad (OMIE) realiza el proceso de casación. Es decir, agrega las ofertas de compra y venta hasta obtener el punto de equilibrio tal y como se muestra en la Figura I. Este punto marca el precio al que pagarán y cobrarán todos los agentes del mercado. 
Uso del role playing en la enseñanza de mercados eléctricos: ejercitar y comprender el poder de mercado en los mismos.

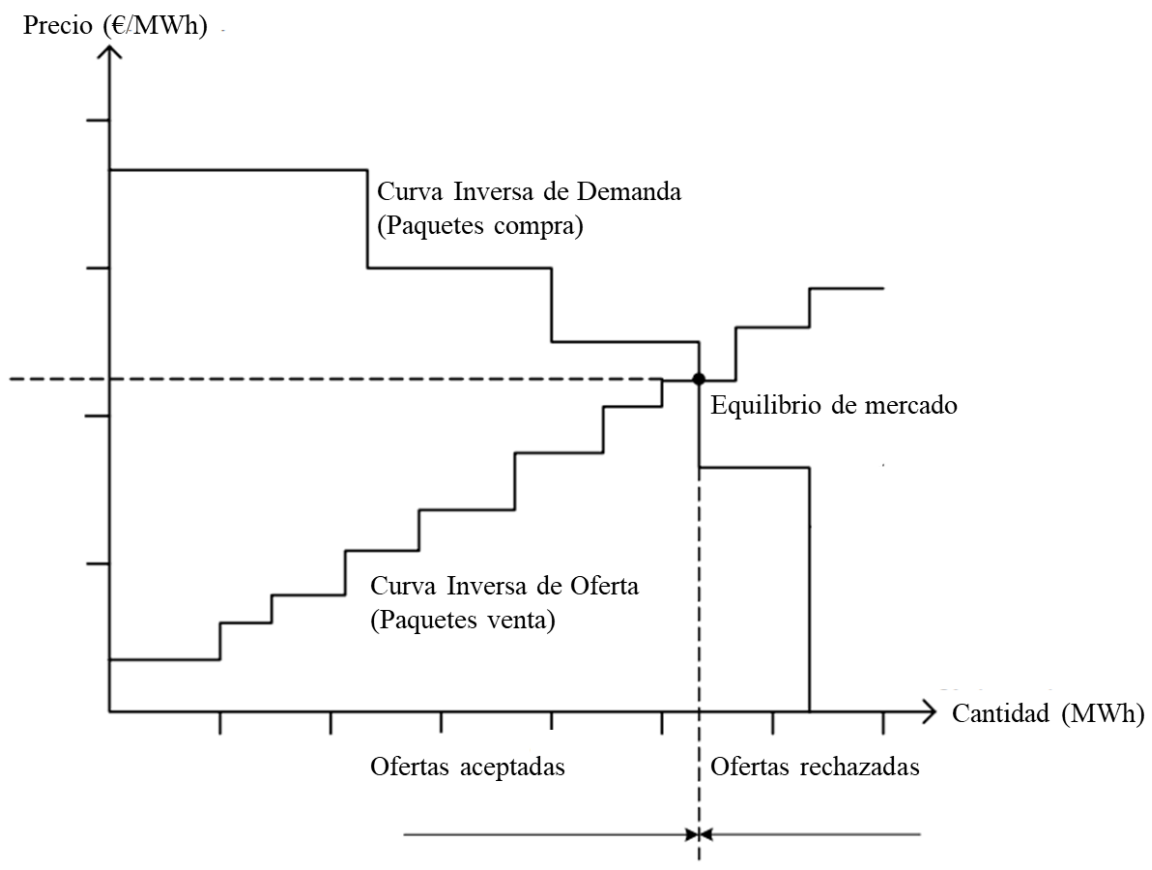

Figura I Casación mercado eléctrico

\section{Excedentes de los consumidores y productores}

A partir del punto de equilibrio se obtiene un precio de mercado, los consumidores que valoraban el producto por encima del precio de equilibrio obtienen la electricidad pagando únicamente el precio de equilibrio, por lo que obtienen un ahorro, el sumatorio de estos ahorros se le denomina excedente neto de los consumidores. De la misma forma para los productores, estos ven incrementados sus beneficios por vender su producto a un precio superior al coste de producción. Al conjunto de estos beneficios se le denomina excedente de los productores. La suma de ambos conceptos se denomina bienestar social y es el elemento que se pretende maximizar con la implantación de mercados competitivos. 


\section{Poder de mercado}

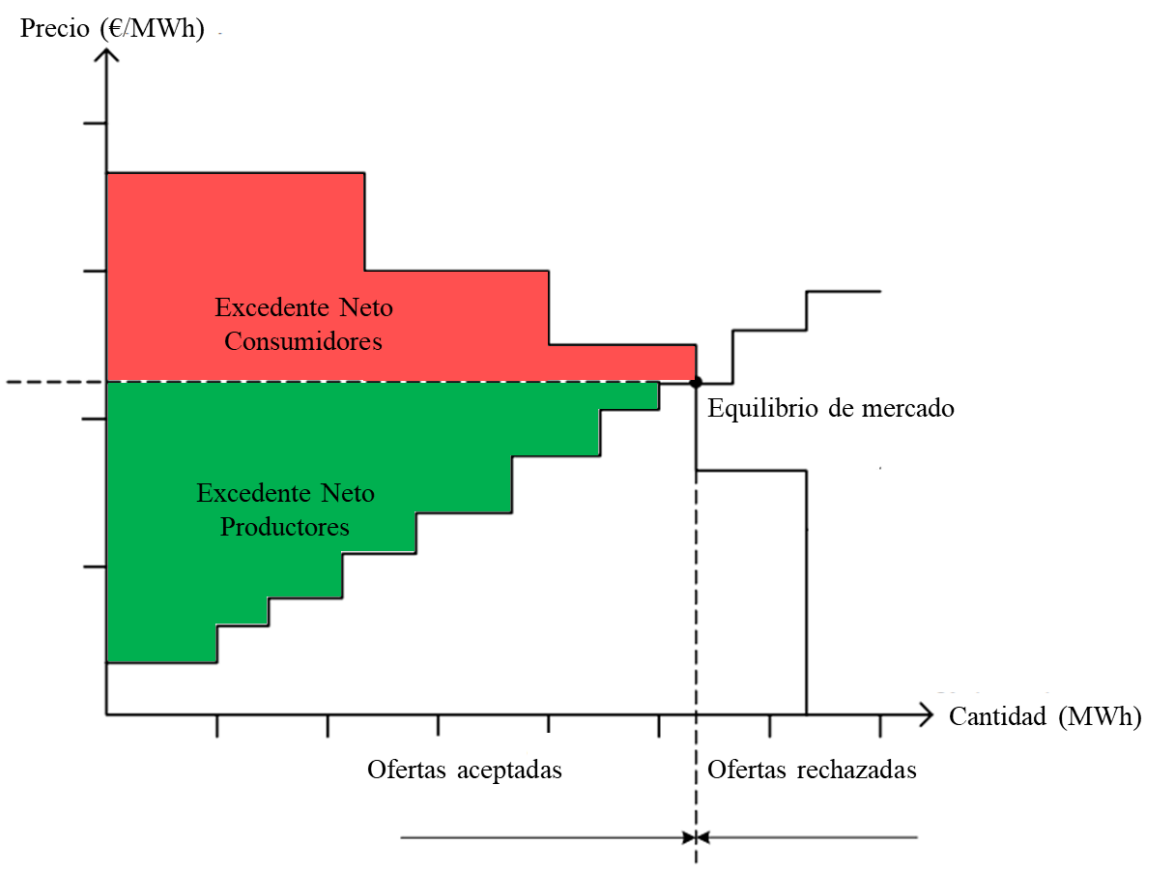

Figura II Excedentes netos de compradores y vendedores.

El poder de mercado es considerado como la capacidad de alterar los precios por encima de los niveles competitivos para aumentar los beneficios individuales Esto ocurre cuando empresas controlan grandes proporciones del mercado o elementos estratégicos del mismo (Ciarreta, Nasirov, \& Silva, 2016). Uno de los índices más comúnmente utilizados para analizar es el índice $\mathrm{HHI}^{1}$, un índice que como muestra la Figura III asciende hasta 1.112,46 en el mercado español. A pesar que esto podría considerarse unos niveles al borde de la competición perfecta, lo cierto es que son las unidades estratégicas las que resultan clave a la hora de argumentar que existe poder de mercado, como es el caso de la hidráulica (Fabra \& Llobet, 2018).

\footnotetext{
${ }^{1}$ Índice Herfindhal-Hirschmann (HHI) se obtiene a través de la suma de los cuadrados de las cuotas de mercado de cada una de las empresas que participan en el mercado.
} 
Uso del role playing en la enseñanza de mercados eléctricos: ejercitar y comprender el poder de mercado en los mismos.

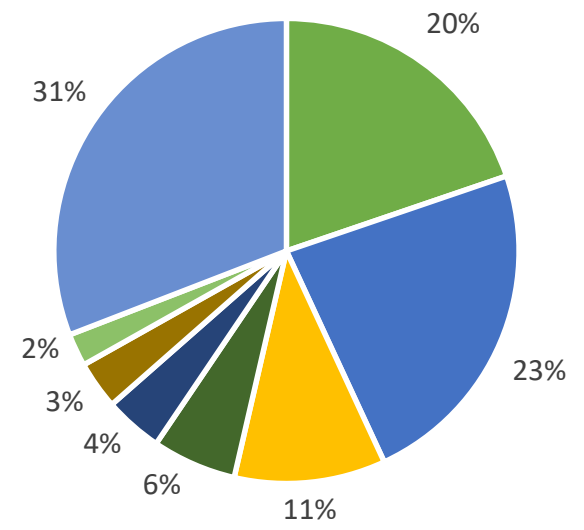

- Iberdrola

- Endesa

Gas Natural

- Acciona

- Hidrocantabrico

- Viesgo

Axpo

- Resto

Figura III Cuotas de las diferentes empresas en la venta de electricidad en España durante 2017. Elaboración propia basado en (OMIE, 2017).

\section{Comportamiento del cártel}

En la realidad, cuando un cártel actúa de forma conjunta para pactar precios y aumentar los beneficios existe la tentación de violar los acuerdos para individualmente aumentar los beneficios a costa del resto de miembros. Cuando una empresa reducir los precios, "traición" al cártel, esta será capaz de colocar en el mercado mayor cantidad de producto que las empresas con las que había acordado, por lo que aumentará su beneficio a corto plazo pero romperá la estabilidad del mismo a largo plazo (Varian, 2017).

\subsection{Plataforma online}

En la plataforma se participa en calidad de comprador o de vendedor de electricidad, tal y como se describe en (Valencia Salazar, 2011). A su vez, la propia plataforma ejerce de gestora de todas las ofertas realizadas por ambos grupos de agentes.

\section{Compradores}

Los agentes que desempeñen el papel de compradores accederán a la ventana que les muestra las características de potencia-coste de sustitución de cada una de las cargas que le han sido asignadas (ver Figura IV). En cada etapa del experimento se envían al Operador de Mercado ofertas dúplex de energía (potencia-precio) para cada unidad de carga. Cada oferta puede ser diferente en precio y potencia, y sólo se comprará por un precio igual o inferior al señalado en la oferta. 


\section{COMPRADOR}

\section{PANEL DE COMPRA DE ENERGÍA}

\begin{tabular}{|c|c|c|c|c|}
\hline Unidad & Potencia (MW) & Valoración (€/MW) & $\begin{array}{c}\text { Potencia a } \\
\text { vender (MW) }\end{array}$ & $\begin{array}{c}\text { Precio de venta } \\
(€ / M W)\end{array}$ \\
\hline Carga 1 & 2.900 & 150 & & \\
\hline Carga 2 & 2.700 & 150 & & \\
\hline Carga 3 & 2.300 & 40 & & \\
\hline Carga 4 & 1.000 & 25 & & \\
\hline Carga 5 & 1.000 & 15 & & \\
\hline
\end{tabular}

Figura IV Pantalla de ofertas del comprador

\section{Vendedores}

Los agentes que desempeñen el papel de vendedores pueden visualizar las características de potencia y coste de producción de cada uno de las unidades generadoras que le han sido asignadas (ver figura 6). En cada etapa del experimento se envían al Operador de Mercado ofertas dúplex de energía (potencia-precio) para cada unidad generadora. Cada oferta puede ser diferente en precio y potencia, y sólo se venderá por un precio igual o superior al señalado en la oferta.

\section{VENDEDOR}

\section{PANEL DE VENTA DE ENERGÍA}

\begin{tabular}{|c|c|c|c|c|}
\hline Unidad & Potencia (MW) & ) Valoración (€/MW) & $\begin{array}{c}\text { Potencia a } \\
\text { vender (MW) }\end{array}$ & $\begin{array}{c}\text { Precio de venta } \\
(€ / \mathrm{MW})\end{array}$ \\
\hline Generador 1 & 3.000 & 10 & & \\
\hline Generador 2 & 3.200 & 11 & & \\
\hline Generador 3 & 3.100 & 20 & & \\
\hline Generador 4 & 2.200 & 36 & & \\
\hline Generador 5 & 2.200 & 42 & & \\
\hline
\end{tabular}

\section{Proceso de casación}

La plataforma recoge todas las ofertas de compradores y vendedores. Una vez recibidas todas las ofertas duales de los diferentes agentes se aplica el método simplex para maximizar el beneficio social, lo cual es equivalente a encontrar el punto de intersección entre la curva agregada de demanda y la de curva agregada de generación. 
Uso del role playing en la enseñanza de mercados eléctricos: ejercitar y comprender el poder de mercado en los mismos.

$$
\max : \sum_{1}^{i} \pi_{i}^{C} * q_{i}^{C}-\sum_{1}^{j} \pi_{j}^{V} * q_{j}^{V}
$$

Siendo $\pi_{i}^{C}$, el precio de la oferta $i$ de compra, $q_{i}^{C}$ la cantidad de la oferta $i$ de compra, $\pi_{j}^{V}$ el precio de la $j$ de venta y $q_{j}^{V}$ la cantidad de la oferta $j$ de venta. Además, el problema de maximización está sujeto a las siguientes restricciones:

$$
\begin{gathered}
\pi_{i}^{C}, q_{i}^{C}, \pi_{j}^{V}, q_{j}^{V} \geq 0 \\
\sum_{1}^{i \in \text { O.Casadas }} q_{i}^{C}-\sum_{1}^{j \in \text { O.Casadas }} q_{j}^{V}
\end{gathered}
$$

La descripción gráfica de los procesos que implica el desarrollo del experimento se puede observar en la figura siguiente:

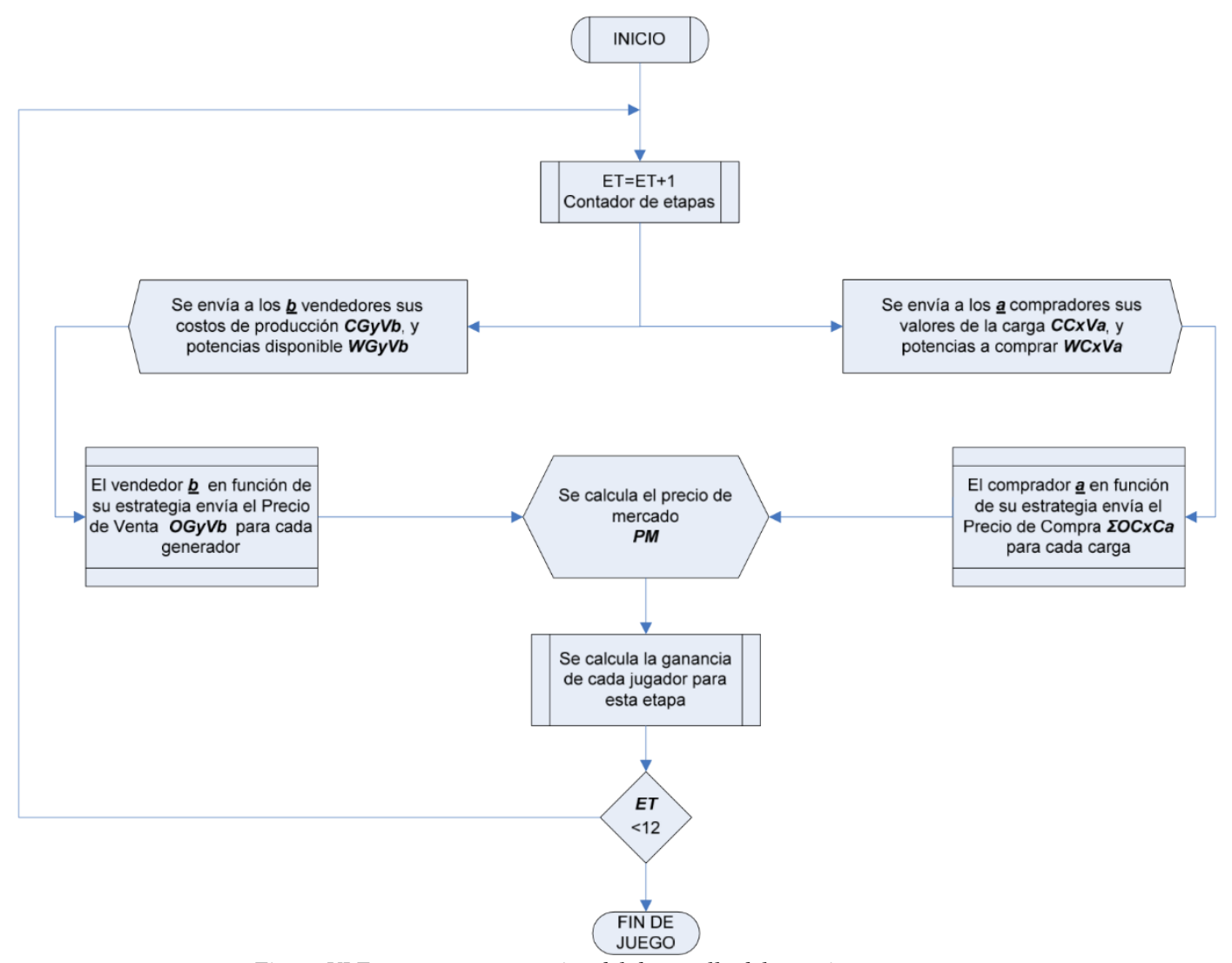

Figura VI Esquema representativo del desarrollo del experimento.

\subsection{Role playing}

La experimentación se desarrolla en un laboratorio web. A este se accede a través de un ordenador por cada participante o grupo de participantes. En él, los sujetos desempeñarán su papel de generador o consumidor que les sea asignado. 
Durante a la práctica se indica al alumnado que existirán 3 casos a la hora de participar en el mercado. En la primera forma, los vendedores tendrán la capacidad de comunicarse entre ellos para pactar precios. En la segunda, serán los consumidores quien puedan ponerse de acuerdo. Finalmente, el tercer caso ningún agente podrá comunicarse con el resto.

\section{Colusión en la oferta}

En este caso se plantea que los vendedores pueden comunicarse entre ellos mismos para tratar de acordar el mayor precio posible e incrementar los beneficios como mejor se crea conveniente. De esta forma, el grupo de vendedores forman un cártel que pacta precios. Por su lado, los consumidores no disponen de ningún canal de comunicación y se comportan como en el caso de mercado competitivo, intentan maximizar sus beneficios realizando ofertas de compra cerca de los beneficios marginales de sus cargas.

\section{Colusión en la demanda}

En este caso se plantea de forma análoga al anterior, lo único que son los compradores los que se comunican para tratar de reducir los precios. De esta forma, el grupo de consumidores forman un cártel de demanda que pacta precios de sus ofertas. Por su lado, los vendedores no disponen de ningún canal de comunicación y se comportan como en el caso de mercado competitivo, intentan maximizar sus beneficios ofertando cerca de los costes marginales de sus generadores.

\section{Mercado competitivo}

En estos mercados, cada agente busca la maximización de su excedente sin tener contacto con otros agentes no la posibilidad de afectar al precio de mercado. Todos los agentes son precio aceptante. Por ello los agentes realizan sus ofertas de compra y venta a un precio asociado a su coste de producción en el caso de los vendedores, y precio asociado a su coste de sustitución en el caso de los compradores.

\section{Resultados y discusión}

La práctica se realizó en grupos de 15 a 20 alumnos. Se van a presentar los resultados de 3 de ellas debido a que representan unos comportamientos característicos que se repitieron en otros casos. Así, nos encontramos diferentes tipos de comportamientos por parte de los participantes. En cada práctica se realizaron 15 etapas de un mismo mercado. Se inició con la colusión en la oferta ( 5 etapas, excepto en el tercer caso que fueron 6), se continuó con colusión en la demanda ( 5 etapas, excepto en el tercer caso que fueron 6) y se finalizó con el mercado de competencia perfecta (5 etapas, excepto en el tercer caso que fueron 3 ).

\section{Caso 1}

En el primer caso se puede observar como el cártel formado por los vendedores comenzó a aumentar los precios de forma drástica (hasta los 70€/MW). Sin embargo, debido a falta de comunicación o distribución desigual de los beneficios, puede observarse como tras la etapa 3 , entre los vendedores se produce una "traición" que genera una tendencia a disminuir el 
Uso del role playing en la enseñanza de mercados eléctricos: ejercitar y comprender el poder de mercado en los mismos.

precio hasta el precio competitivo. Como puede observarse, esto ocurrió también en el caso del cártel de la demanda, tras una bajada de precios hasta los $13 € / \mathrm{MW}$, ciertos compradores no comparten la distribución de beneficios, saliéndose de los pactos y tendiendo de nuevo hacia el precio de competencia perfecta. Finalmente, durante las etapas de competencia perfecta el precio se mantuvo de forma constante en los $26 € / \mathrm{MW}$.

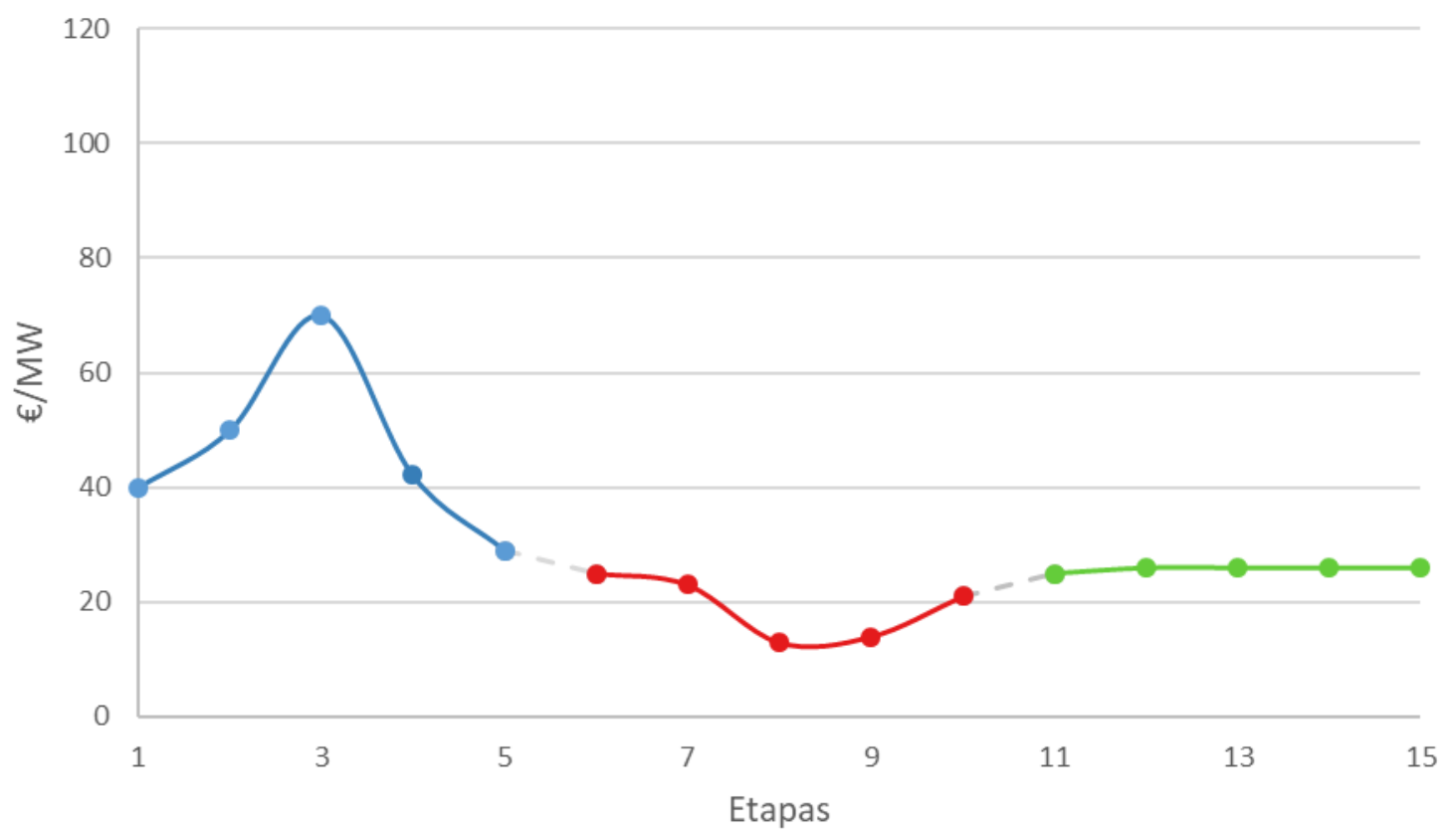

$\longrightarrow$ Colusión oferta $\quad \longrightarrow$ Colusión demanda $\quad \longrightarrow$ Competencia perfecta

Figura VII Evolución del precio de mercado, sesión 1.

En este caso, se puede observar como una comunicación deficiente o un reparto injusto de los beneficios dentro de los acuerdos genera "traiciones" y la pérdida de poder de mercado para aumentar los precios y salir beneficiado como conjunto de agentes.

\section{Caso 2}

El segundo caso demuestra claramente como el cártel de vendedores fue capaz de ponerse de acuerdo y aumentar sistemáticamente los precios de la electricidad hasta los $100 € / \mathrm{MW}$. A diferencia del caso anterior, se puede observar que no existieron "traiciones" dentro del cártel y que la subida de precios fue respaldada por todos los miembros del mismo, consiguiendo así aumentar los beneficios que obtenían los generadores. Cuando inició la etapa del cártel de os compradores, el precio se redujo rápidamente hasta niveles 5 veces inferiores. Sin embargo, estos no ejercieron su poder de mercado y los precios se mantuvieron de forma casi constante en estos niveles. Finalmente, se puede observar como cuando las etapas de competencia perfecta comenzaron, el precio subió hasta estabilizarse alrededor de los 27 $€ / M W$ con pequeñas fluctuaciones entre etapas. 


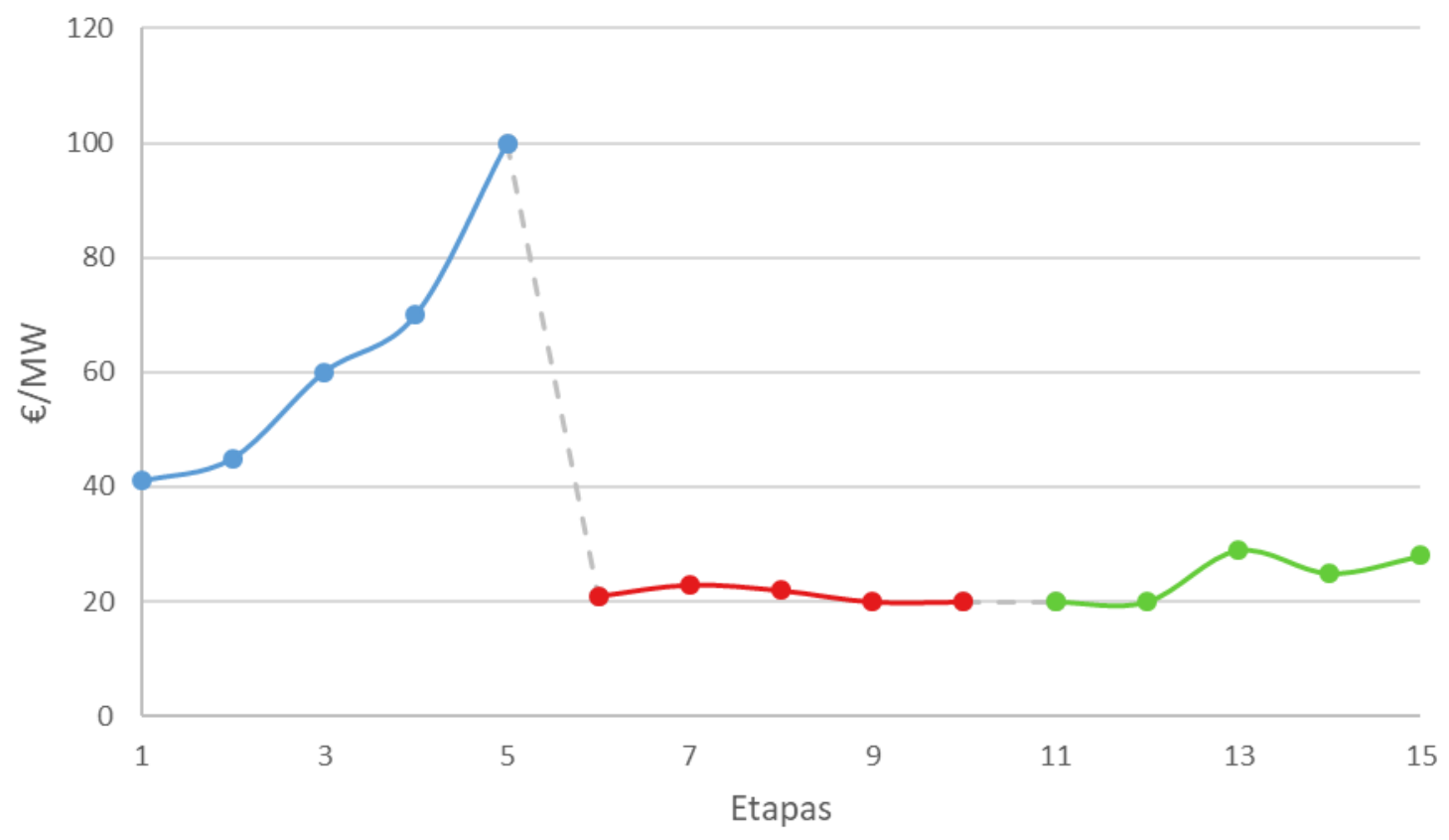

$\multimap$ Colusión oferta $\rightarrow$ Colusión demanda $\quad \longrightarrow$ Competencia perfecta

Figura VIII Evolución del precio de mercado, sesión 2.

En este caso, se puede observar como una comunicación óptima y un reparto a priori justo de los beneficios dentro de los acuerdos genera que el cártel siga compacto. Además, en este caso se pudo comprobar una política muy agresiva de subida de precios que fructiferó proporcionando elevados beneficios a los vendedores.

\section{Caso 3}

En el tercer caso se puede observar como durante la primera fase de cártel, los vendedores fueron aumentando el precio progresivamente con etapas de consolidación. Si bien no hubo ningún caso de "traición" al mismo, como ocurrió en el caso 1, se puede observar como la política fue mucho menos agresiva que en el caso 2 , llegando únicamente a un precio máximo de $45 € / M W$. Durante la segunda etapa, los consumidores fueron capaces de ir bajando progresivamente el precio de mercado, pero este siempre se encontró por encima del precio de mercado. Esto da a entender que los consumidores no fueron capaces de ponerse de acuerdo en realmente presionar los precios a la baja tal y como eran capaces. Esto se ve en las últimas etapas de competencia perfecta donde el precio se estabilizó sobre los $20 € / \mathrm{MW}$.

En este caso, se puede observar como una comunicación eficiente es capaz de ir aumentando progresivamente el precio de mercado produciendo un beneficio a los vendedores. Sin 
Uso del role playing en la enseñanza de mercados eléctricos: ejercitar y comprender el poder de mercado en los mismos.

embargo, puede observarse como los compradores no fueron capaces de utilizar su capacidad de disminuir el precio para beneficiarse, ya que únicamente neutralizaron poco a poco el aumento de precios que habían ocasionado los vendedores.

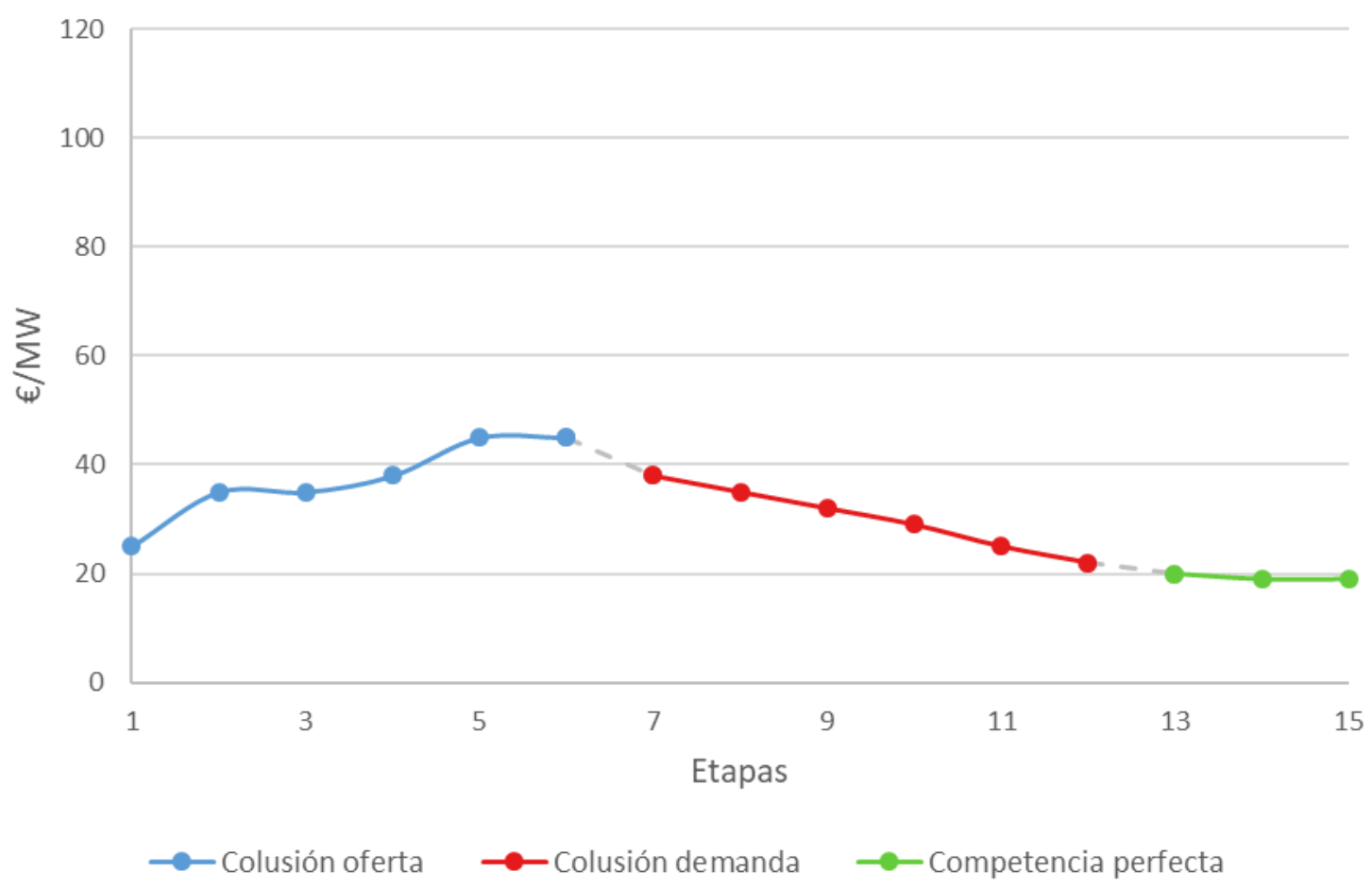

Figura IX Evolución del precio de mercado, sesión 3.

En los diferentes casos que ocurrieron en diferentes sesiones se pudo comprobar la capacidad que tienen los diferentes agentes para modificar el mercado en beneficio propio. Es relevante contextualizar que por la regulación existente en el mercado eléctrico los generadores tienen una capacidad muy superior de utilizar su poder de mercado. Esto puede verse en como los precios de la electricidad aumentaron hasta niveles de los $100 € / \mathrm{MW}$ cuando eran los vendedores lo que colusionaban, mientras que cuando el cártel era formado por los compradores el precio únicamente bajó a $13 € / \mathrm{MW}$. Es relevante mencionar que los precios de mercado competitivo rondaban los $25 € / \mathrm{MW}$, por lo que mientras los generadores los pudieron aumentar hasta aun $300 \%$, los consumidores únicamente pudieron reducirlos en un $50 \%$. Estos comportamientos tienen elevados impactos en la distribución de beneficios y el bienestar de consumidores y productores.

La práctica también ayudó a comprender las diferentes actitudes que puede tomar un cártel, desde mantener pactos estables, a aumentar los precios continuamente hasta a que existieran "traiciones" dentro del mismo para beneficiarse individualmente. 


\section{Feedback estudiantes}

A pesar de la falta de datos para realizar análisis estadísticos, el alumnado ha demostrado mucho interés en la aplicación de juegos de rol en el campo de los mercados energéticos, comentando la práctica como una de las mejores actividades de la asignatura. Basados en los comentarios de los estudiantes y la entrega de prácticas, se percibe que la mayor parte de los estudiantes comprendieron los elementos básicos el funcionamiento del mercado eléctrico y las complejidades asociadas a mercados imperfectos con un grado de poder de mercado.

Algunas de las reacciones de los estudiantes fueren las siguientes:

- "Me pareció una práctica divertida. Confirmó la facilidad que tendrían las empresas proveedoras a formar un cartel si no interviniera el estado. También, se pudo entender mejor cómo funciona el procedimiento de casación de precio de la electricidad al presentar nosotros en primera persona las ofertas de compra y venta."

- "La práctica es muy entretenida de hacer. Además, es bastante útil para observar los comportamientos básicos de los mercados competitivos: cuan útiles pueden ser los lobbys para el establecimiento de precios, cómo y por qué puede iniciarse una guerra de precios... etc."

Estos resultados van en concordancia con otros proyectos similares realizados en otras universidades de ingeniería eléctrica que han utilizado metodologías similares para la comprensión de los mercados eléctricos. Entre otras conclusiones, Naukkarinen et al. (2017) mencionan que el alumnado no únicamente desarrolla competencias profesionales aplicadas, sino que también les ayuda a reflexionar y comprender mejor conceptos complejos asociados a la materia. Por su parte, Carpio et al. (2007) refuerza la idea de la idoneidad para motivar y desarrollar competencias del alumnado.

\section{Conclusiones}

La experiencia llevada a cabo ha tenido una muy buena respuesta, tanto a nivel de adquisición de competencias como de aceptación por parte del alumnado. El uso de metodologías de role playing permite comprender conceptos técnicos de una manera práctica. Es por ello, que el uso del mismo junto a herramientas que simulan el mercado eléctrico permite al alumnado comprender como funcionan los mismos en entornos sin competencia perfecta. Mediante la combinación de ambas se consigue la adquisición no solamente de competencias prácticas, sino también de conceptos complejos a nivel económico que son experimentados por el propio alumnado. Tal y como se ha descrito, las prácticas de laboratorio fueron un éxito y reflejaron los diferentes casos que pueden ocurrir en la formación de cárteles empresariales y de consumidores. 
Uso del role playing en la enseñanza de mercados eléctricos: ejercitar y comprender el poder de mercado en los mismos.

\section{Referencias}

Alcázar Ortega, M., \& Álvarez Bel, C. (2018). Utilización de Microsoft EXCEL en la enseñanza de sistemas eléctricos de potencia: desarrollo de un método matricial para la resolución del problema de despacho económico. In Libro de Actas IN-RED 2018: IV Congreso Nacional de Innovación Educativa y Docencia en Red (pp. 1077-1090). Valencia: Universitat Politècnica València. https://doi.org/10.4995/INRED2018.2018.8562 Borenstein, S., Bushneil, J., \& Knittel, C. R. (1999). Market Power in Electricity Markets: Beyond Concentration Measures. The Energy Journal1, 20(4), 65-88.

Borenstein, S., Bushnell, J., Kahn, E., \& Stoft, S. (1995). Market power in California electricity markets. Utilities Policy, 5(3-4), 219-236. https://doi.org/10.1016/09571787(96)00005-7

Carpio, J., Quejo, G., Guirado, R., Valcarcel, M., Simon, P., Santamaria, A., ... FernandezPerez, M. D. (2007). Educational Application of Role-Playing and Simulation of Professional Environments Related to the Power Systems and the Electricity Market. In 2007 IEEE Meeting the Growing Demand for Engineers and Their Educators 2010-2020 International Summit (pp. 1-10). IEEE. https://doi.org/10.1109/MGDETE.2007.4760380

Ciarreta, A., Nasirov, S., \& Silva, C. (2016). The development of market power in the Spanish power generation sector: Perspectives after market liberalization. https://doi.org/10.1016/j.enpol.2016.06.029

CNMC. Resolución del procedimiento sancionador incoado a Iberdrola Generación, S.A.U. por manipulación fraudulenta tendente a alterar el precio de la energía mediante el incremento de las ofertas de las unidades de gestión hidráulica de Duero, Sil y Tajo (2015).

Contreras, J., Conejo, A. J., de la Torre, S., \& Munoz, M. G. (2002). Power Engineering Lab: Electricity Market Simulator. IEEE Power Engineering Review, 22(2), 60-60. https://doi.org/10.1109/MPER.2002.4311996

Fabra, N., \& Llobet, G. (2018). A propósito de la reciente subida del precio de la luz. Nada Es Gratis. Retrieved from http://nadaesgratis.es/gerard-llobet/a-proposito-de-la-recientesubida-del-precio-de-la-luz

Fabra, N., \& Toro, J. (2005). Price wars and collusion in the Spanish electricity market. International Journal of Industrial Organization, 23(3-4), 155-181. https://doi.org/10.1016/J.IJINDORG.2005.01.004

Guevara-Cedeno, J. Y., Palma-Behnke, R., \& Uribe, R. (2012). Experimental Economics for Teaching the Functioning of Electricity Markets. IEEE Transactions on Education, 55(4), 466-473. https://doi.org/10.1109/TE.2012.2189886

Hua, D., Ngan, H. ., \& Fushuan Wen. (2007). Electricity market simulator: Adaptive for studies on different market behavior. In TENCON 2007 - 2007 IEEE Region 10 Conference (pp. 1-5). IEEE. https://doi.org/10.1109/TENCON.2007.4428852 
Montes Granada, W. F. (2004). Prácticas de laboratorio en ingeniería: una estrategia efectiva de aprendizaje - Willler Ferney Montes Granada - Google Libros. In Notas Universitarias,. Ibagué, Colombia.

Naukkarinen, J., Niemela, H., Makkonen, M., Valtonen, P., Tikka, V., Tarkkala, T., ... Belonogova, N. (2017). Development of a second-generation power exchange game by applying design science. In 2017 19th European Conference on Power Electronics and Applications (EPE'17 ECCE Europe) (p. P.1-P.10). IEEE.

https://doi.org/10.23919/EPE17ECCEEurope.2017.8099319

OMIE. (2017). Informes mensuales | OMIE. Retrieved March 7, 2019, from http://www.omie.es/inicio/publicaciones

OMIE. (2019). Nuestros mercados de electricidad | OMIE. Retrieved March 13, 2019, from http://www.omie.es/inicio/mercados-y-productos/mercado-electricidad/nuestros-mercadosde-electricidad

Valencia Salazar, I. (2011). Diseño e implementación de una metodología de subasta iterativa aplicada a mercados eléctricos competitivos. Riunet. Universitat Politècnica de València, Valencia (Spain). https://doi.org/10.4995/Thesis/10251/13258

Varian, H. R. (2017). Microeconomía intermedia : un enfoque actual. 